\title{
NONLINEAR MODEL PREDICTIVE CONTROL OF ROBOTS, CRANES AND VEHICLES
}

\author{
Béla Lantos, Bálint Kiss
}

Department of Control Engineering and Informatics, Budapest University of Technology and Economics Magyar Tudósok krt. 2, H-1117 Budapest,Hungary

\begin{abstract}
The main goal of the paper is to show that nonlinear model based predictive control is an effective alternative method for controlling uncertain nonlinear underactuated systems satisfying real time expectations where uncertainty is caused by friction and disturbance. The paper presents control algorithms for a two degree of freedom robot and for two underactuated systems, the small size model of a planar crane and a weeled mobile robot, which are based on nonlinear model predictive control. The continuous time dynamic model has been discretized and the finite dimensional optimization problem is solved by conjugate gradient technique in every horizon. Extended Kalman filter is used for state and disturbance estimation. For the crane the initial approximation of the control sequence within the actual horizon is determined by using the flatness properties. For development purposes a multiprocessor system has been elaborated where the control algorithm is running under QNX real-time operating system. The paper also presents experimental results that demonstrate the applicability of the proposed algorithms under real time conditions. Copyright (C) 2005 IFAC
\end{abstract}

Keywords: Predictive control, Nonlinear systems, Robotics, Disturbance rejection, Realtime systems

\section{INTRODUCTION ${ }^{1}$}

Model predictive control is a popular method especially in the process industry where relatively slow process models allow online optimization. Linear predictive control is well elaborated both in frequency (operator) domain (Camacho, E. F. and C. Bordons, 2000; Lantos, 2003) and state space (Rossiter et al., 1998; Lantos, 2003). Depending on the type of constraints optimal prediction leads to Quadratic (QP) or Nonlinear Programming (NP) wich are well supported by existing softwares (e.g. Optimization Toolbox in MATLAB).

\footnotetext{
${ }^{1}$ Support for the research was provided by the Hungarian National Research Programs under grant No. OTKA T042634, NKFP 2/016/2001 and NKTH RET 04/2004 and by the Control System Research Group of the Hungarian Academy of Sciences.
}

For nonlinear systems recent methods are usually based on new optimum seeking methods suited for the predictive control problem or traditional analytical optimum conditions and gradient based optimization techniques (Allgöver and Zheng, 2000; Kim and Shin, 2003). Basis for the later ones is a general form of the Lagrange multiplicator rule which is also valid in Banach spaces (Lantos, 2003). Typical finite horizon nonlinear predictive control problems in discrete time lead to optimization in finite dimensional space where the variables are $x=\left\{x_{i}\right\}_{i=0}^{N}$ and $u=\left\{u_{i}\right\}_{i=0}^{N-1}$, the optimality criterion is

$$
\begin{aligned}
F_{0}(x, u) & =\sum_{i=0}^{N-1}\left[<Q_{i} x_{i}, x_{i}>+<R_{i} u_{i}, u_{i}>\right] / 2 \\
& +<Q_{N} x_{N}, x_{N}>/ 2 \\
& =: \sum_{i=0}^{N-1} L_{i}\left(x_{i}, u_{i}\right)+\Phi\left(x_{N}\right),
\end{aligned}
$$


the constraints are the state equation

$$
\varphi\left(x_{i}, u_{i}\right)-x_{i+1}=0,
$$

control set $u_{i} \in M$ and initial condition $a-x_{0}=0$.

If $\left(x^{*}, u^{*}\right)$ is the optimal solution then

is the derivative of

$$
f(x, u)=J_{x}^{\prime}\left(x^{*}, u^{*}\right) x+J_{u}^{\prime}\left(x^{*}, u^{*}\right) u
$$

$$
\begin{aligned}
J(x, u)= & F_{0}(x, u)+<\lambda_{0}, a-x_{0}>+<\lambda_{1}, \varphi\left(x_{0}, u_{0}\right)-x_{1}> \\
& +\cdots+<\lambda_{N}, \varphi\left(x_{N-1}, u_{N-1}\right)-x_{N}>.
\end{aligned}
$$

By introducing the Hamiltonian functions as

$$
H_{i}=<\lambda_{i+1}, \varphi\left(x_{i}, u_{i}\right)>+L_{i}\left(x_{i}, u_{i}\right),
$$

the necessary condition of the optimality results in

$$
\begin{gathered}
\lambda_{N}=Q_{N} x_{N}, \\
\lambda_{i}=\partial H_{i} / \partial x_{i}=Q_{i} x_{i}+\left(\partial \varphi / \partial x_{i}\right)^{T} \lambda_{i+1}, \\
\partial H_{i} / \partial u_{i}=R_{i} u_{i}+\left(\partial \varphi / \partial u_{i}\right)^{T} \lambda_{i+1}, \\
d J=\sum_{i=0}^{N-1}<\partial H_{i} / \partial u_{i}, u_{i}^{*}-u_{i}>\leq 0 .
\end{gathered}
$$

For the control design within the actual horizon first the initial condition $x_{0}$ and the approximation of $u$ are needed (the latter may be the solution in the previous horizon shifted to the left).

The optimization repeats the following steps:

1. Solution of the state equations in $x=\left\{x_{i}\right\}_{i=0}^{N}$.

2. Computation of the Lagrange multiplicators $\lambda_{i}$.

3. Computation of the derivatives $\partial H_{i} / \partial u_{i}$.

4. Numerical optimization based on gradient type methods (gradient, conjugate gradient,

Davidon-Fletcher-Powell etc.) to find

$$
u=\left\{u_{i}\right\}_{i=0}^{N-1} \text {. }
$$

Non-predictive design method should be used to find the initial approximation for the first horizon. If the original sytem is a continuous time one then first it can be approximated by a discrete time one, e.g.

$\dot{x}=f_{c}(x, u) \Rightarrow x_{i+1}=x_{i}+T f_{c}\left(x_{i}, u_{i}\right)=: \varphi\left(x_{i}, u_{i}\right)$

where $T$ is the sampling time. If the full state can not be measured then $x_{0}$ can be approximated by using extended Kalman filter.

If $y=C x$ is the system output and $\tilde{y}=y_{d}-y$ is the error then the cost function can be modified as

$$
\begin{gathered}
\tilde{y}=y_{d}-C x, \\
2 L_{i}\left(x_{i}, u_{i}\right)=<\tilde{Q}_{i} \tilde{y}_{i}, \tilde{y}_{i}>+<S_{i} x_{i}, x_{i}>+<R_{i} u_{i}, u_{i}>, \\
2 \Phi\left(x_{N}\right)=<\tilde{Q}_{N} \tilde{y}_{N}, \tilde{y}_{N}>,
\end{gathered}
$$

and the derivatives can be computed by

$$
\begin{gathered}
\partial L_{i} / \partial x_{i}=-C^{T} \tilde{Q}_{i} \tilde{y}_{i}+S_{i} x_{i}, \\
\partial \Phi / \partial x_{N}=-C^{T} \tilde{Q}_{N} \tilde{y}_{N} .
\end{gathered}
$$

Input constraints are enforced by projecting $u_{i}$ into the constraints set. State constraints can be taken into consideration as an additional penalty added to $L\left(x_{i}, u_{i}\right)$ in the cost function. It is known that the weighting in the term $\Phi\left(x_{N}\right)$ has great influence on the stability and dynamic behaviour of the sytem under predictive control (Allgöver and Zheng, 2000). To improve the stability properties the techniques of Frozen Riccati Equation (FRE) and Control Lyapunov Function (CLF) can be suggested (Yu et al., 2001). Unfortunately their real time realization is time consuming. In the paper experimentally chosen weighting terms have been applied.

The control design strategy can be summarized in the following steps:

1. Development of the nonlinear dynamic model of the system.

2. Optimal (suboptimal, flatness-based etc.) open loop control signal design used for initial approximation of the control sequence in the horizon.

3. Identification of the nonlinear friction model.

4. Elaboration of the disturbance model reduced on the system input.

5. Development of the model based nonlinear predictive controller and its use in closed loop control. The first element of the control sequence in the actual horizon is completed by the feedforward compensations of the friction and the disturbance.

\section{NONLINEAR PREDICTIVE CONTROL OF A 2-DOF ROBOT ARM}

A two degree of freedom (DOF) open chain rigid robot having nonlinear friction effect $h_{f}(q, \dot{q})$ and dynamic model $H(q) \ddot{q}+h(q, \dot{q})+h_{f}(q, \dot{q})=\tau \quad$ is considered first. Low level torque (current) control is assumed hence the control input is the torque $\tau$. Inertia and friction parameters in the model are the results of previously performed identification and assumed known. The dynamic model of the robot arm (without the friction effect) is

$$
\begin{gathered}
D_{11} \ddot{q}_{1}+D_{12} \ddot{q}_{2}+2 D_{121} \dot{q}_{1} \dot{q}_{2}+D_{122} \dot{q}_{2}^{2}+D_{1}=\tau_{1} \\
D_{12} \ddot{q}_{1}+D_{22} \ddot{q}_{2}-D_{112} \dot{q}_{1}^{2}+D_{2}=\tau_{2},
\end{gathered}
$$

where the parameters and functions are

$$
\begin{aligned}
& \alpha_{1}=m_{1} l_{c 1}^{2}+m_{2}\left(l_{1}^{2}+l_{c 2}^{2}\right)+I_{1}+I_{2} \\
& \alpha_{2}=m_{2} l_{1} l_{c 2} \\
& \alpha_{3}=m_{2} l_{c 2}^{2}+I_{2} \\
& \alpha_{4}=g\left(m_{1} l_{c 1}+m_{2} l_{1}\right) \\
& \alpha_{5}=g m_{2} l_{c 2} \\
& D_{11}=\alpha_{1}+2 C_{2} \alpha_{2} \\
& D_{12}=\alpha_{3}+C_{2} \alpha_{2} \\
& D_{22}=\alpha_{3} \\
& D_{112}=-S_{2} \alpha_{2}=D_{122} \\
& D_{1}=C_{1} \alpha_{4}+C_{12} \alpha_{5} \\
& D_{2}=C_{12} \alpha_{5}
\end{aligned}
$$

and $C_{12}=\cos \left(q_{1}+q_{2}\right)$ etc. as usual in robotics. In the experiments $m_{1}=m_{2}=5, \quad I_{1}=I_{2}=1$, $l_{1}=l_{2}=1, l_{c 1}=l_{c 2}=0.5$ have been used (all in SI units). If $x=\left(q^{T}, \dot{q}^{T}\right)^{T}$ denotes the state then the 
nontrivial part of the state equation is $\ddot{q}=-H^{-1} h+H^{-1} \tau$.

The predictive control algorithm needs the derivative of the right side of the state equation by $x$ and $u$. Meanwhile it can be utilized that if $f(x)=A^{-1}(x) b(x)$ is any smooth function then

$$
\frac{\partial f}{\partial x_{i}}=-A^{-1} \frac{\partial A}{\partial x_{i}} A^{-1} b+A^{-1} \frac{\partial b}{\partial x_{i}}
$$

The robot repeats a predefined path which allows that the desired $q_{d}(t+k)$ and $\dot{q}_{d}(t+k)$ are known in the prediction horizon. The initial approximation for the first horizon is based on the gravity compensation in the initial state and the compensation of the friction. Unknown disturbance (load etc.) consisting of first order deterministic polynomial and Gaussian noise was considered on the input of the robot (output of the controller). Experiments with estimated $\dot{q}$ and estimated disturbances $d_{1}, d_{2}$ reduced on the system inputs are performed. For estimation of the augmented state extended Kalman filter was used (Chui and Chen, 1999; Lantos, 2003).

Optimization is based on conjugate gradient technique. For horizon length $N=10$ satisfactory accuracy of the optimization can be reached within $T=25 \mathrm{~ms}$ sampling time on standard processors. Fig. 1 shows the results. If needed, fine interpolation in closed loop is also possible based on $u(t)$ and $u(t+1)$ determined in the actual horizon. For large $N$ basis functions (splines etc.) can be suggested.
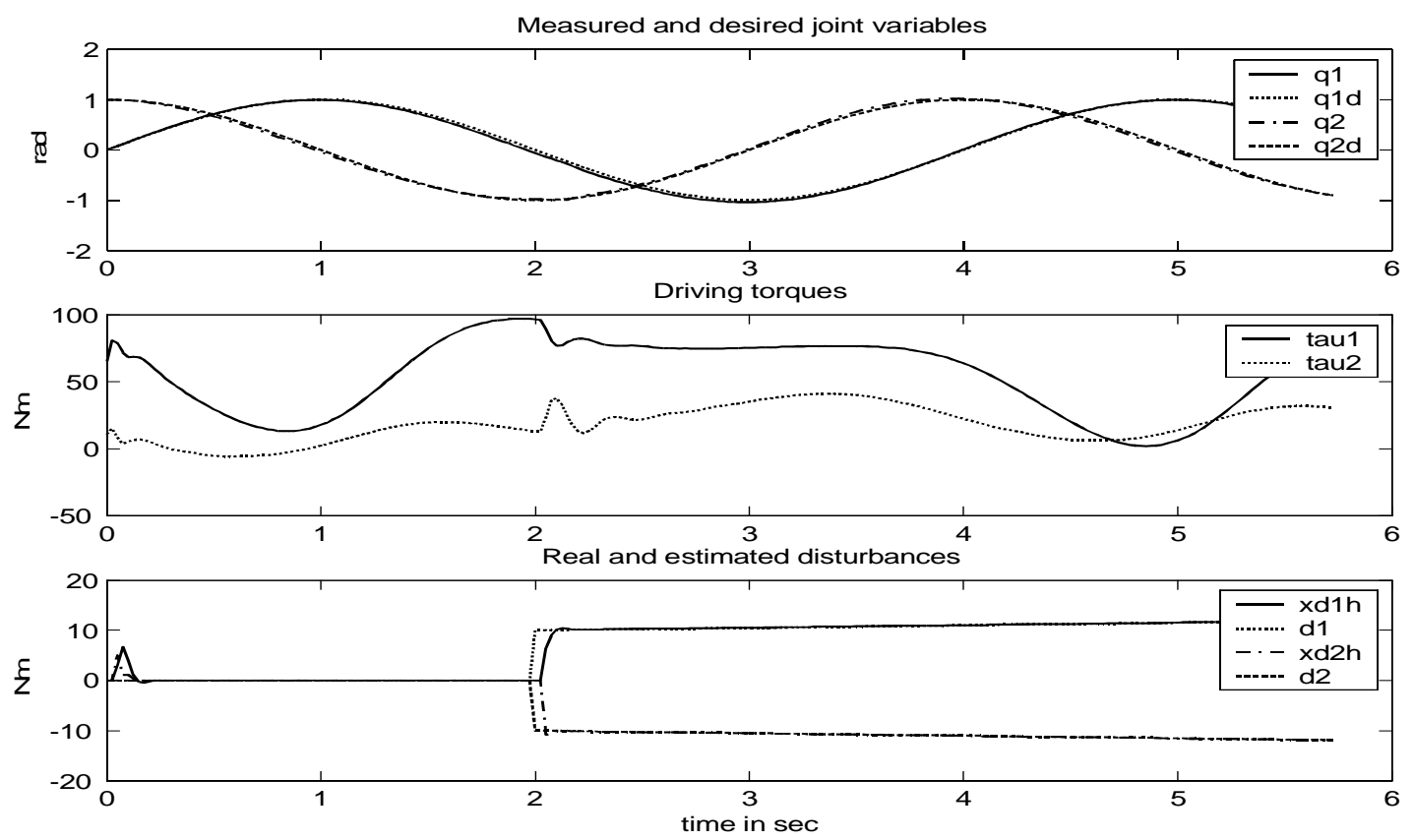

Fig. 1. Behaviour of the 2-DOF robot arm during predictive control.

\section{NONLINEAR PREDICTIVE CONTROL OF A 2D CRANE}

The second problem is the control of the small size model of a real cable-driven crane used by US Navy (Kiss et al., 2000). For simplicity only the two dimensional (2D) planar version is considered. The goal is the precise and quick motion of the load without overshoot. The scheme of the $2 \mathrm{D}$ crane is shown in Fig. 2. (Since the pulley's mass in point $B$ has been neglected hence $L_{s}$ and its actuator motor is omitted in the discussion). The planar crane is a 3DOF system which is underactuated because the only inputs are the two motor torques moving the ropes $L_{1}$ and $L_{2}+L_{3}$. The model of the planar crane is differencially flat (Kiss, 2001). In simplified formulation it means that if $\dot{x}=f(x, u)$ is the dynamic model of the system then there exist a new variable $y$ (the flat output) and finite integers $q$ and

$$
\begin{array}{lll}
p \quad \text { such that } \quad x=\phi_{1}\left(y, \dot{y}, \ddot{y}, \ldots, y^{(q)}\right), & \\
u=\phi_{2}\left(y, \dot{y}, \ddot{y}, \ldots, y^{(q+1)}\right), & y=\psi_{1}\left(x, u, \dot{u}, \ldots, u^{(p)}\right) .
\end{array}
$$

For the crane $y:=(x, z)^{T}$ where $x, z$ are the coordinates of the load in the plane of the crane. The flatness relations for the 2D crane are summarized as follows:

$$
\begin{aligned}
& \theta=\arctan (-\ddot{x} /(\ddot{z}+g) \\
& d=\frac{\ddot{x}\left(z-(k+l) C_{\alpha}\right)-(\ddot{z}+g)\left(x-(k+l) S_{\alpha}\right)}{(\ddot{z}+g) S_{a}-\ddot{x} C_{a}} \\
& \beta=\arcsin \left((1-d / l) S_{\alpha+\theta}\right) \\
& \gamma=(\pi+\beta-(\alpha+\theta)) / 2 \\
& L_{1}=l S_{\beta} / S_{\gamma} \\
& L_{2}=l S_{\gamma-\beta} / S_{\gamma} \\
& x_{B}=(k+l) S_{\alpha}-L_{2} S_{\alpha-\beta} \\
& z_{B}=(k+l) C_{\alpha}-L_{2} C_{\alpha-\beta}
\end{aligned}
$$




$$
\begin{aligned}
& L_{3}=\sqrt{\left(x-x_{B}\right)^{2}+\left(z-z_{B}\right)^{2}} \\
& T_{3}=m_{\text {load }}\left((\ddot{z}+g) C_{\theta}-\ddot{x} S_{\theta}\right) \\
& u_{1}=T_{1} \rho_{1}-\frac{J_{1}}{\rho_{1}} \ddot{L}_{1} \\
& u_{2}=T_{3} \rho_{2}-\frac{J_{2}}{\rho_{2}}\left(\ddot{L}_{2}+\ddot{L}_{3}\right)
\end{aligned}
$$

If $y, \dot{y}, \ldots, y^{(4)}$ are known then all system variables can be computed from them except singularities. For prescribed initial $y_{0}$ and final $y_{1}$ and transient time $T_{F}$ five times differenciable $y(t)$ polynomials can be found connecting the points with straight line from which all the system states $x(t)$ and inputs $u(t)$ can be reconstructed. Results $u_{1}, u_{2}$ of the flatness based design have been used for the initial approximation of the sequence $u$ in all the horizons.

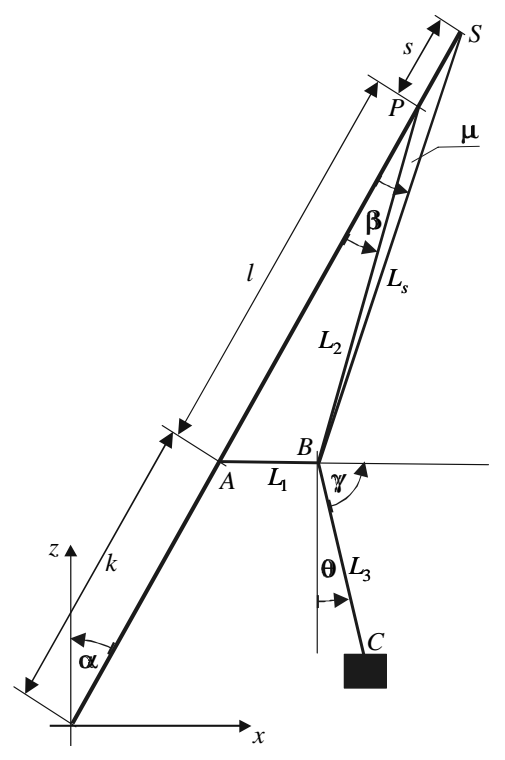

Fig. 2. The scheme of the 2D crane.

It follows from the equilibrium of the internal forces that the line $A B$ in the direction of the horizontal rope $L_{1}$ is the bisectrix of the angle $2 \gamma$ between $P B$ and $B C$. Hence the magnitudes of the internal forces $T_{3}$ for $L_{2}$ and $T_{1}$ for $L_{1}$ are related by $T_{1}=2 C_{\gamma} T_{3}$. By using the flat output all the variables discribing the $2 \mathrm{D}$ crane can be determined by (8). In order to avoid the first and second order symbolic differentiation of the nonlinear expressions for $L_{1}, L_{2}, L_{3}$, numerical differentiation was applied (see spline, unmkpp, mkpp in MATLAB). Finally the flatness based input torques $u_{1}, u_{2}$ for the motors can be determined, where $J_{i}$ and $\rho_{i}$ denote the inertia and the radius of the winch, respectively.

Assuming zero derivatives until order five in the initial and final positions, the $11^{\text {th }}$ order polynomial connecting $\left(x_{0}, z_{0}\right)$ and $\left(x_{1}, z_{1}\right)$ has the form

$$
\begin{aligned}
x(\tau)= & x_{0}+\left(x_{1}-x_{0}\right) \tau^{6} \times \\
& {\left[a_{6}+a_{7} \tau+a_{8} \tau^{2}+a_{9} \tau^{3}+a_{10} \tau^{4}+a_{11} \tau^{5}\right] }
\end{aligned}
$$

where $\tau=t / T_{F}$ is the normalized time and the coefficients for both $x(\tau)$ and $z(\tau)$ are

$$
\begin{array}{lll}
a_{6}=0.462, & a_{7}=-1.980, & a_{8}=3.465, \\
a_{9}=-3.080, & a_{10}=1.386, & a_{11}=-0.252 .
\end{array}
$$

Neglecting the feedforward compensated friction and the centripetal and Coriolis effects (because of the slow angular velocities of typical cranes) the basic equations of the underactuated system are

$$
\begin{gathered}
\frac{J_{1}}{\rho_{1}} \ddot{L}_{1}=T_{1} \rho_{1}-u_{1}, \\
\frac{J_{2}}{\rho_{2}}\left(\ddot{L}_{2}+\ddot{L}_{3}\right)=T_{3} \rho_{2}-u_{2} .
\end{gathered}
$$

The system is submitted to constraints with respect to $L_{1}$ and $L_{2}$ hence Lagrange multiplicators could have been used. Fortunately, instead of this relatively complicated method, the equilibrium between internal forces can be exploited, see (Kiss, 2001), who developed a form of the dynamic model for the choice $\quad x=\left(\gamma, \alpha-\beta, L_{3}, \dot{\gamma},(\dot{\alpha}-\dot{\beta}), \dot{L}_{3}\right)^{T} \quad$ where $\alpha=$ const . However the measured outputs are $L_{1}, L_{2}+L_{3}, \dot{L}_{1}, \dot{L}_{2}+\dot{L}_{3}$ which depend on these state variables in a nonlinear way making necessary the use of nonlinear state estimation and some modifications of the predictive control algorithm of (Kim and Shim, 2003). Hence a new model has been developed using $x=\left(L_{1}, L_{2}, L_{3}, \dot{L}_{1}, \dot{L}_{2}, \dot{L}_{3}\right)^{T}$. The idea is the use of the cosine theorem by which

$$
C_{\gamma}=\frac{l^{2}-L_{1}^{2}-L_{2}^{2}}{2 L_{1} L_{2}}, C_{\beta}=\frac{l^{2}+L_{2}^{2}-L_{1}^{2}}{2 l L_{2}} .
$$

For $L_{1}$ and $L_{2}$ the expressions in (8) can be used. By elementary geometry and using

$$
\begin{gathered}
\varphi=(\pi / 2)-(\gamma+(\alpha-\beta)), \\
\theta=\pi-(2 \gamma+(\alpha-\beta)),
\end{gathered}
$$

it yields

$$
\begin{aligned}
x & =k S_{\alpha}+L_{1} C_{\varphi}+L_{3} S_{\theta} \\
& =k S_{\alpha}+L_{1} S_{\gamma+(\alpha-\beta)}+L_{3} S_{2 \gamma+(\alpha-\beta)}, \\
z & =k C_{\alpha}+L_{1} S_{\varphi}-L_{3} C_{\theta} \\
& =k C_{\alpha}+L_{1} C_{\gamma+(\alpha-\beta)}+L_{3} C_{2 \gamma+(\alpha-\beta)} .
\end{aligned}
$$

The main problem is the computation of $T_{3}$ from the state variables which is needed in (11). This can be performed using $m \ddot{x}=-T_{3} S_{\theta}, m(\ddot{z}+g)=T_{3} C_{\theta}$ for the load, from which it follows

$C_{\theta} \ddot{x}+S_{\theta}(\ddot{z}+g)=0, \quad m\left(-S_{\theta} \ddot{x}+C_{\theta}(\ddot{z}+g)=T_{3}\right.$.

The remaining part is based on carefully performed symbolic differentiation to find the Jacobian $(d x)$ and Hessian $(D 2 x)$ of the necessary functions, for example in the case of $x(t)$

$$
\begin{aligned}
\dot{x}= & d x \cdot\left(\dot{L}_{1} \dot{L}_{2} \dot{L}_{3}\right)^{T}, \\
\ddot{x}= & d x \cdot\left(\ddot{L}_{1} \ddot{L}_{2} \ddot{L}_{3}\right)^{T}+ \\
& \left(\dot{L}_{1} \dot{L}_{2} \dot{L}_{3}\right) \cdot D 2 x \cdot\left(\begin{array}{lll}
\dot{L}_{1} & \dot{L}_{2} & \dot{L}_{3}
\end{array}\right)^{T} .
\end{aligned}
$$

The dynamic model in MATLAB notation is the following: 


$$
\begin{aligned}
& A(1,:)=C_{\theta} d x+S_{\theta} d z \\
& b(1)=\left(\dot{L}_{1} \dot{L}_{2} \dot{L}_{3}\right)\left(-C_{\theta} D 2 x-S_{\theta} D 2 z\right)\left(\dot{L}_{1} \dot{L}_{2} \dot{L}_{3}\right)^{T}-S_{\theta} g, \\
& A(2,:)=2 C_{\gamma} m\left(S_{\theta} d x-C_{\theta} d z\right) \rho_{1}, \\
& A(2,1)=A(2,1)+J_{1} / \rho_{1}, \\
& b(2)=\left(\dot{L}_{1} \dot{L}_{2} \dot{L}_{3}\right)\left(2 C _ { \gamma } m \left(-S_{\theta} D 2 x+\right.\right. \\
& \left.\left.\quad C_{\theta} D 2 z\right) \rho_{1}\right)\left(\dot{L}_{1} \dot{L}_{2} \dot{L}_{3}\right)^{T}+2 C_{\gamma} m C_{\theta} g \rho_{1}, \\
& A(3,:)=m\left(S_{\theta} d x-C_{\theta} d z\right) \rho_{2}, \\
& A(3,2: 3)=A(3,2: 3)+\left[J_{2} / \rho_{2} J_{2} / \rho_{2}\right] \\
& b(3)=\left(\dot{L}_{1} \dot{L}_{2} \dot{L}_{3}\right)\left(m \left(-S_{\theta} D 2 x+\right.\right. \\
& \left.\left.\quad C_{\theta} D 2 z\right) \rho_{2}\right)\left(\dot{L}_{1} \dot{L}_{2} \dot{L}_{3}\right)^{T}+m C_{\theta} g \rho_{2} \\
& \left(\ddot{L}_{1} \ddot{L}_{2} \ddot{L}_{3}\right)^{T}=A^{-1} b-A^{-1}\left(0 u_{1} u_{2}\right)^{T} .
\end{aligned}
$$

Unfortunately, for predictive control $A$ and $b$ have to be differentiated once more, see (3).

An important question is the appropriate choice of the sampling time $T$ so that the approximation of the continous time system by the discrete time one in (4) is accurate enough. For this purpose a stright line motion of the load is designed by using the above flatness technique resulting in the open loop control signals $u_{1}(t)$ and $u_{2}(t)$ and the flatness based rope length and load position. The parameters of the small size 2D crane model were $\alpha=0.4454 \mathrm{rad}$, $k=0.1662 \mathrm{~m}, \quad l=0.351 \mathrm{~m}, \quad J_{1}=J_{2}=10^{-4} \mathrm{~kg} / \mathrm{m}^{2}$ and $\rho_{1}=\rho_{2}=0.025 \mathrm{~m}$. It was experimentally proved that for $u_{1}(t), u_{2}(t)$ and $T=0.002 \mathrm{~s}$ the discrete time approximation reproduces the results $L_{1}(t), L_{2}(t), L_{3}(t)$ determined by the flatness technique. It is clear that the small sampling time is the consequence of the small size model (1:80 reduction) and for real crane much larger sampling time is possible.

The optimization has been performed by using conjugate gradient technique. Satisfactory accuracy of the optimisation has been reached within reasonable time, see Fig. 3, however it is far away of the real time expectations in the case of the small size model.
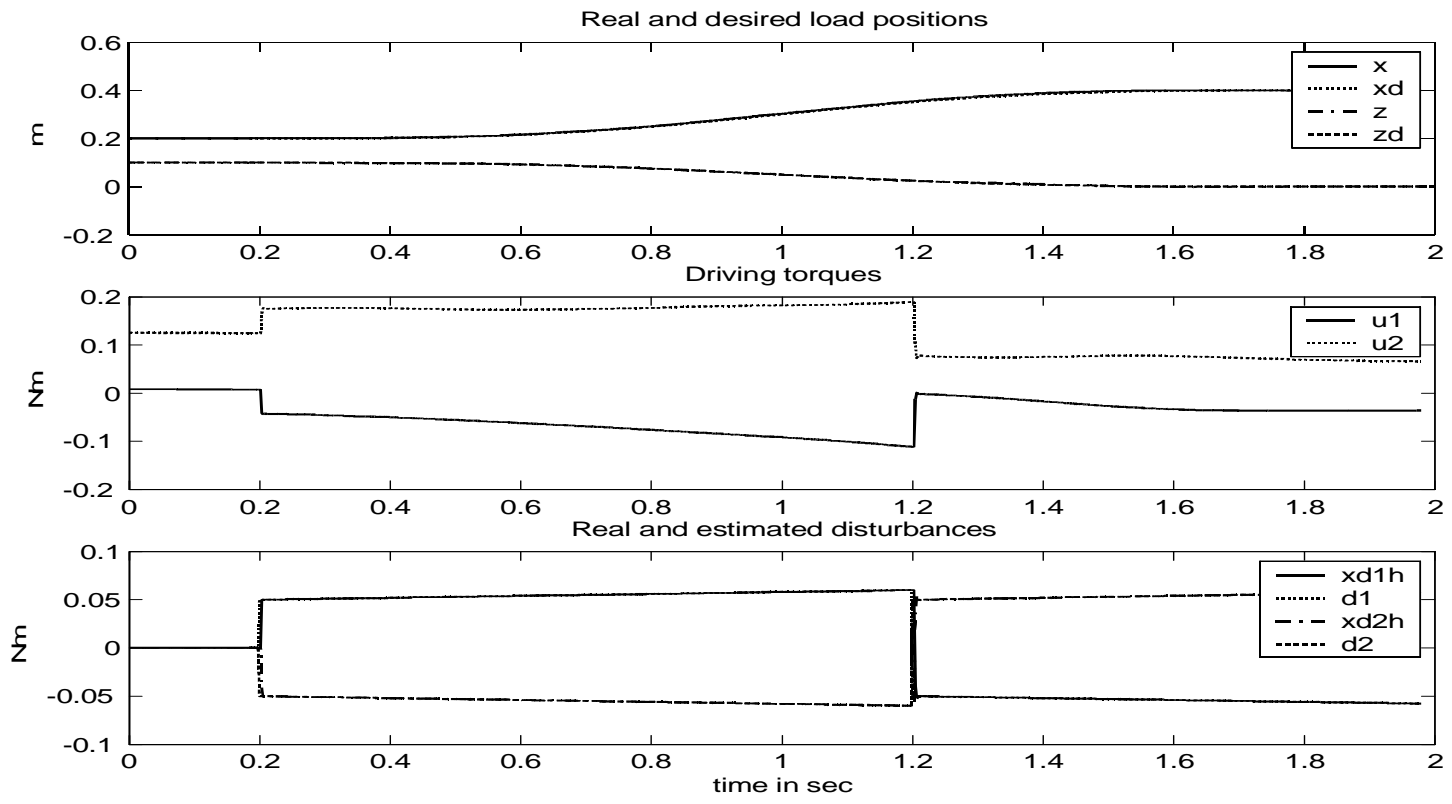

Fig. 3. Behaviour of the small size model of the 2D crane during predictive control.

\section{PREDICTIVE CONTROL OF A WHEELED MOBILE ROBOT}

The third problem, which will be investigated in the paper, is the control of a wheeled mobile robot. It is known (Warren et al., 2001) that both position control and tracking can be performed by using nonlinear time variable dynamic state feedback. Nonlinear predictive control is an alternative method for both type of problems.

The control is divided into 3 levels. High level control designs the motion of the reference mobile robot in the form $\dot{x}_{r}=v_{r} \cos \vartheta_{r}, \dot{y}_{r}=v_{r} \sin \vartheta_{r}$ and $\dot{\vartheta}_{r}=\omega_{r}$. Low level control realizes the control of the mobile robot according to the control outputs of the middle level based on the kinematic model. Only the high and middle levels have been investigated.

State, output and input are $x:=(x, y, \vartheta)^{T}, y:=x$ and $u:=(v, \omega)^{T}$, respectively. The system model is $f_{c}(x, u)=\left(u_{1} \cos x_{3}, u_{1} \sin x_{3}, u_{2}\right)^{T}$.

The derivatives $\partial f_{c} / \partial x_{i}, \partial f_{c} / \partial u_{i}$ needed for NPC can easily be determined. $T=0.02 s$ is suitable for discrete time approximation of the continuous time system if the speed is in the order of $0.5 \mathrm{~m} / \mathrm{s}$. Horizon length $N=10$ has been chosen. The reference robot's $u:=\left(v_{r}, \omega_{r}\right)^{T}$ is used in NPC as initial approximation of the control sequence in the first horizon. Constant plus linear disturbances reduced on the mobile robot inputs have been assumed and estimated by using extended Kalman 
filter. Optimization has been performed by using conjugate gradient technique. Satisfactory accuracy of the optimization has been reached within reasonable time fulfilling real time expectations, see Fig. 4.
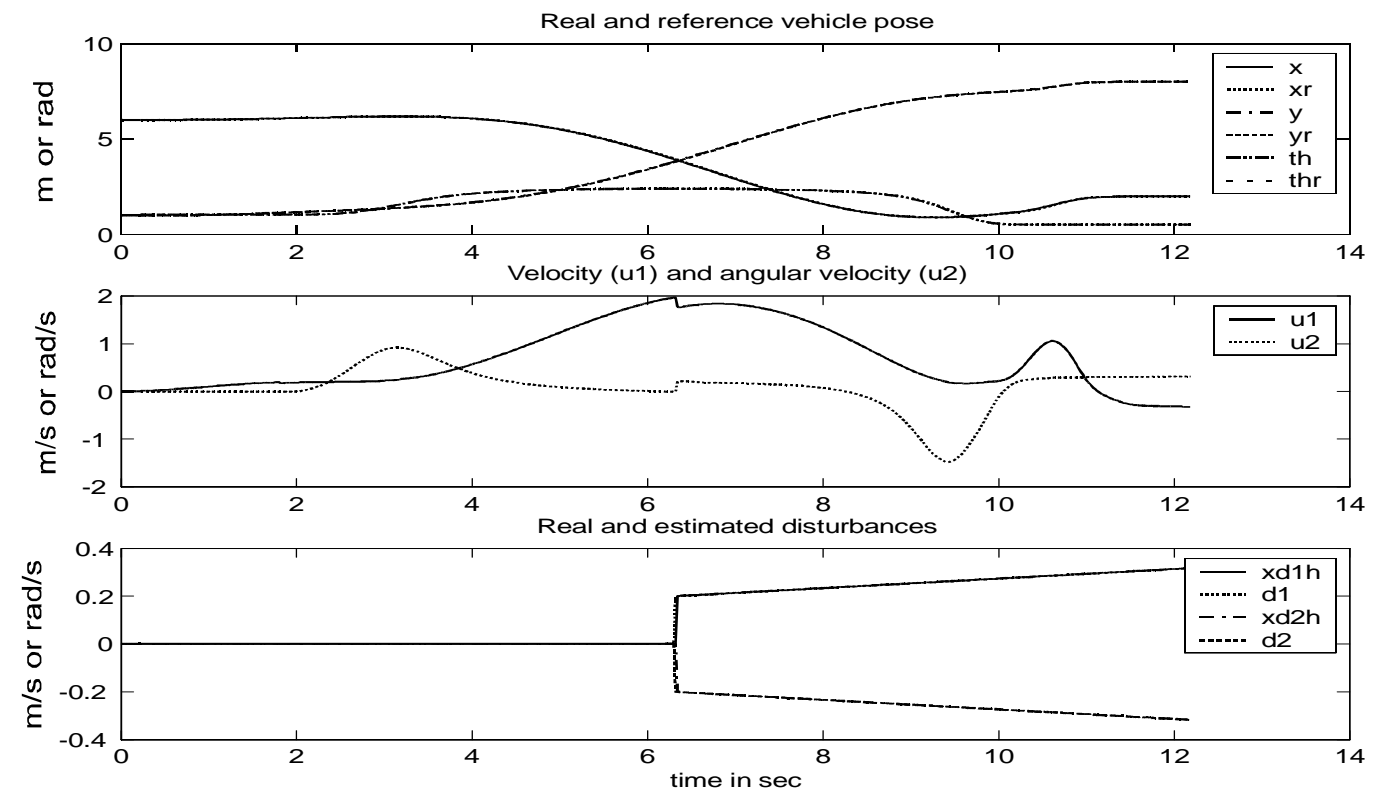

Fig. 4. Behaviour of the mobile robot during predictive control.

\section{CONCLUSIONS}

The experiments were performed on a multiprocessor system consisting of three computers. On the first computer runned the NPC control and the extended Kalman filter under QNX real-time operating system. Another process managed the receipt of the sensory information and the sending of the controller outputs to the system. The actual system (robot arm, crane or mobile robot) was simulated as separate QNX process on the first computer. This process communicated with the other parts by using messages. The simulated system can easily be substituted in future experiments by real systems saving the whole controller architecture. On the second computer (running also under QNX) were performed the off-line design of the reference signals for the robot arm, the flatness based path and driving torque design for the crane and the design of the path of the reference mobile robot, respectively. The third computer runned under Windows2000 and documented the real control, state and output information in graphical form.

The results in the three examples show that nonlinear predictive control can be effectively used not only in the process control but also in the control of uncertain moving systems including fully actuated robots, and underactuated cranes and vehicles. Nonlinear predictive control is able to improve the control performances and can take into consideration the real time system constraints. The time needed for the convergence of the online numerical optimization can be guaranteed for $T=25 \mathrm{~ms}$ sampling time and $N=10 T$ horizon length in case of standard processors and QNX real time operating system. If needed, further fine interpolation can be applied.

These give real chance also for applications in more complicated fields like robot cooperation, formation motion of aircraft systems and cooperation between underground and aerial vehicles.

\section{REFERENCES}

Camacho, E. F. and C. Bordons (2000). Model predivtive control. Springer, London.

Rossiter, J.A. et al. (1998). A numerically robust state-space approach to stable-predictive control strategies. Automatica, 34, 65-73.

Lantos, B. (2003). Control systems theory and design. Vol. II. Advanced control systems (in Hungarian). Hungarian Academic Press, Budapest.

Allgöwer,F. and A. Zheng ed. (2000). Nonlinear predictive control. Birkhäuser, Basel.

Kim, H.J. and D. H. Shim (2003). A flight control system for aerial robots. Control Engineering Practice, 11, 1389-1400.

$\mathrm{Yu}$, J. et al. (2001). Comparison of nonlinear control design techniques on a model of the Caltech ducted fan. Automatica, 37, 1971-1978.

Chui, C.K. and G. Chen (1999). Kalman filtering with real-time applications. Springer, Berlin.

Kiss, B., J. Levine and Ph. Mullhaupt (2000). Control of a reduced size model of US Navy crane. In Isidori: Nonlinear control of the year 2000, Springer, London.

Kiss, B. (2001). Motion planning and control of a class of flat and Liouvillian mechanical systems. $\mathrm{PhD}$ Thesis, L'Ecole Nationale Superieure des Mines de Paris.

Warren, E.D. et al. (2001). Nonlinear control of wheeled mobile robots. Springer, London. 